\title{
Chimie des matériaux hybrides
}

\section{Clément Sanchez}

\section{(2) OpenEdition}

Journals

Édition électronique

URL : https://journals.openedition.org/annuaire-cdf/12934

DOI : 10.4000/annuaire-cdf.12934

ISBN : 978-2-7226-0490-2

ISSN : 2109-9227

Éditeur

Collège de France

Édition imprimée

Date de publication : 1 novembre 2016

Pagination : 229-248

ISBN : 978-2-7226-0453-7

ISSN : 0069-5580

Référence électronique

Clément Sanchez, "Chimie des matériaux hybrides », L'annuaire du Collège de France [En ligne], 115 |

2016, mis en ligne le 22 juin 2018, consulté le 19 décembre 2022. URL : http://

journals.openedition.org/annuaire-cdf/12934 ; DOI : https://doi.org/10.4000/annuaire-cdf.12934 


\title{
Chimie des matériaux hybrides
}

\author{
M. Clément SANCHEZ, membre de l'Institut \\ (Académie des sciences), professeur
}

\section{ENSEIGNEMENT}

\section{Cours. Interfaces : science des matériaux, biologie, médecine II ${ }^{\mathrm{a}}$}

Cette année nous avons consacré un ensemble de leçons à l'analyse des interfaces entre les nanoparticules (NP) et les biocomposantes et à l'étude des méthodologies permettant d'optimiser ces interfaces et de «camoufler» les bio-nano-objets afin d'optimiser leur efficacité dans des traitements thérapeutiques ou cosmétiques. Sur la base à la fois de caractérisations structurales et physico-chimiques des nanoparticules et de leur activité in vitro et in vivo, une analyse détaillée de la toxicité ou de la biocompatibilité de NP d'oxydes métalliques et de silices a été présentée.

Un second ensemble de leçons a été consacré à l'étude des diatomées, des algues phytoplanctoniques dont les formes extraordinairement belles émerveillent aussi bien les artistes que les scientifiques. Leur description structurale, l'étude des processus de biominéralisation et la présentation de leurs nombreuses propriétés, source d'applications dans les domaines des matériaux fonctionnels, de l'énergie et de l'environnement, ont fait l'objet des deux dernières leçons. Ces deux dernières leçons inaugurent un cours sur les biominéraux, leur processus de formation et leurs propriétés qui sera poursuivi l'an prochain. Les titres des six leçons sont:

- l'interface biologie-nano-objets ;

- camouflage et vecteurs thérapeutiques hybrides ;

- oxydes métalliques et stress oxydant;

- $\mathrm{SiO}_{2}$ en milieu biologique : faisons un point ;

- diatomées : belles et utiles ;

- diatomées : de la dynamite au cristal photonique.

a. Le cours est disponible en audio et en vidéo sur le site internet du Collège de France : http://www.college-de-france.fr/site/clement-sanchez/course-2014-2015.htm [NdÉ]. 


\section{L'interface biologie-nano-objets et le camouflage des vecteurs thérapeutiques}

La croissance rapide des nanotechnologies augmente la probabilité de contact entre les nanomatériaux et les êtres humains et leur environnement. Les nanoparticules (NP) peuvent interagir avec les protéines, les membranes, les cellules, l'ADN des organites. Il est donc important d'établir une meilleure compréhension de l'interface entre les nanoparticules et les composantes biologiques. Ces interfaces nanoparticules / composantes biologiques dépendent des forces colloïdales ainsi que de nombreuses interactions biophysicochimiques dynamiques. Ces interactions conduisent à la formation de couronnes de protéines qui enveloppent les particules entrainant ainsi des modifications des phénomènes d'absorption intracellulaire et des procédés biocatalytiques. Ces modifications peuvent avoir des effets biologiques indésirables ou modifier la biocompatibilité. Pour leur part, les biomolécules peuvent induire des transformations de phase, la restructuration et la dissolution de la surface des nanomatériaux. Sonder et analyser ces différentes interfaces et leur dynamique permet le développement de relations prédictives entre la structure et l'activité des NP. Ces relations sont déterminées par les propriétés des nanomatériaux telles que la taille, la forme, la chimie de surface, la rugosité et les revêtements présents sur leur surface. Il est très important d'établir de façon fiable cette connaissance car elle doit permettre une utilisation plus sûre des nanomatériaux.

C'est dans ce cadre, aux interfaces entre les nanomatériaux et les systèmes biologiques, que les mondes bio-organiques et synthétiques se fondent dans une nouvelle science, qui étudie non seulement les paramètres permettant une utilisation sécurisée des nanotechnologies mais aussi la conception de nanomatériaux sur mesure pour des applications biologiques. L'interface «nano-bio » comprend les interactions physico-chimiques dynamiques, la cinétique et la thermodynamique des échanges de matière entre les surfaces de nanomatériaux et les surfaces des composants biologiques (protéines, membranes, phospholipides, vésicules d'endocytose, d'autres organelles, l'ADN et les l'ensemble des fluides biologiques). Pour permettre à ce domaine d'évoluer, nous devons comprendre les forces dynamiques et les composants moléculaires qui façonnent ces interactions. Il est aujourd'hui encore impossible de décrire avec certitude toutes les interactions biophysicochimiques mises en jeu à l'interface «nano-bio ». Cependant, l'unification des connaissances partielles générées par les différentes disciplines doivent nous fournir un cadre conceptuel permettant de guider cette exploration. Dans ce cadre, nous explorons ces interfaces du point de vue des forces élémentaires qui régissent la chimie colloïdale et les adaptations qui se produisent au niveau des interfaces biologiques. Après la mise en suspension de nanoparticules dans un tissu ou dans un milieu biologique, et après interaction avec des cellules (surfaces des membranes des compartiments endosomiques, des organelles et du cytoplasme), nous analysons les interfaces nanoparticules-biocomposantes et discutons des méthodes qui permettent de sonder l'interface «nano-bio». Déterminer les interactions des processus tels que la formation de la couronne de protéines, le contact cellulaire, l'emballage des particules à la surface des cellules, l'endocytose et la biocatalyse intracellulaire, et sonder ces différentes interfaces exigent des idées et des techniques d'imagerie nouvelles. Mieux comprendre ces mécanismes doit nous permettre de prendre des décisions plus éclairées sur les modes de conception des nano-objets.

Après avoir évalué plus d'une centaine de familles de nanoparticules différentes, y compris des fullerènes, des oxydes métalliques, des polymères, des liposomes, des 
dendrimères, des boîtes quantiques et des colloïdes d'or, il semble que l'hydrophobie, la taille et la charge de surface sont les principaux paramètres influençant la biocompatibilité des nanoparticules.

In vivo, les temps de demi-vies des nanoparticules hydrophobes sont généralement de courte durée (quelques secondes à quelques minutes) parce qu'elles sont rapidement capturées et retirées de la circulation par les éléments cellulaires du système réticulo-endothélial, pour aboutir principalement dans le foie et dans la rate. Bien que ce problème puisse limiter la biodisponibilité et l'utilité des nanoparticules en tant que vecteurs de principes actifs, certaines applications in vivo reposent sur une absorption rapide par les macrophages pour transporter les nanoparticules vers les ganglions lymphatiques ou d'autres sites de l'inflammation (par exemple pour l'imagerie par résonance magnétique du foie par des nanoparticules de fer paramagnétiques). L'importance de la taille des particules est corroborée par des données révélant que les poumons, les voies gastro-intestinales et la peau sont des obstacles très efficaces contre l'absorption et la diffusion de nanoparticules. Par exemple, les oxydes métalliques et les nanoparticules de polystyrène ne pénètrent pas dans la peau humaine saine, tandis que les quantum dot (boîtes quantiques) peuvent pénétrer la peau endommagée et se loger dans d'autres tissus et organes. Lorsque les nanoparticules sont délibérément injectées dans la circulation sanguine, leur taille a un effet important sur les taux et les voies de dégagement du corps. Par exemple, les reins ne peuvent excréter des particules de taille inférieure à $8 \mathrm{~nm}$. D'autre part, via des macrophages spécialisés, le foie et la rate peuvent piéger des particules de taille supérieure à $200 \mathrm{~nm}$. Bien que le foie puisse a priori capturer toute particule dont la taille est supérieure à environ $200 \mathrm{~nm}$, la clairance de la circulation sanguine ne signifie pas nécessairement l'excrétion, car la voie biliaire permet seulement d'éliminer des nanoparticules de diamètre inférieur à $30 \mathrm{~nm}$. Les cellules de Kupffer retiennent les particules jusqu'à ce que le foie puisse les dégrader. Les nanoparticules de tailles comprises entre $30 \mathrm{~nm}$ et quelques centaines de nanomètres peuvent subir une accumulation passive sur le site d'une tumeur grâce à la pénétration accrue associée à l'effet EPR (Enhanced Permeability and Retention). Ce phénomène se réfère à la perméabilité accrue de la vascularisation des tumeurs, qui, lorsqu'elle est couplée avec un drainage lymphatique diminué, permet d'accumuler les nanomatériaux au voisinage du système vasculaire cancéreux peu étanche. Enfin, l'importance de la charge de particules a été illustrée par des études in vivo et in vitro d'une série de nanoparticules de taille relativement constante faisant varier leur charge de surface en signe et en valeur absolue. Les particules cationiques sont plus cytotoxiques et plus susceptibles d'induire l'agrégation des hématies et des plaquettes que les nanoparticules neutres ou anioniques. Cette tendance est confirmée par des études de la capacité des particules de polystyrène cationiques à induire une cytotoxicité, des fuites vasculaires et des infiltrations inflammatoires dans les poumons de rats et de souris. Ce mécanisme de toxicité peut aussi expliquer l'apparition d'œdèmes et de bronchiolites pulmonaires aiguës chez les humains exposés à la peinture constituée d'aérosols à base de nanoparticules cationiques.

Cette analyse peut être résumée et illustrée à l'aide d'un diagramme de phase en trois dimensions proposé par Scott McNeil [1,2] (figure 1). Ce diagramme résume parfaitement les principales tendances qualitatives de la biocompatibilité des nanoparticules révélées après le criblage in vivo d'environ 130 nanoparticules destinées à des utilisations variées, en particulier comme vecteurs en biologie et 


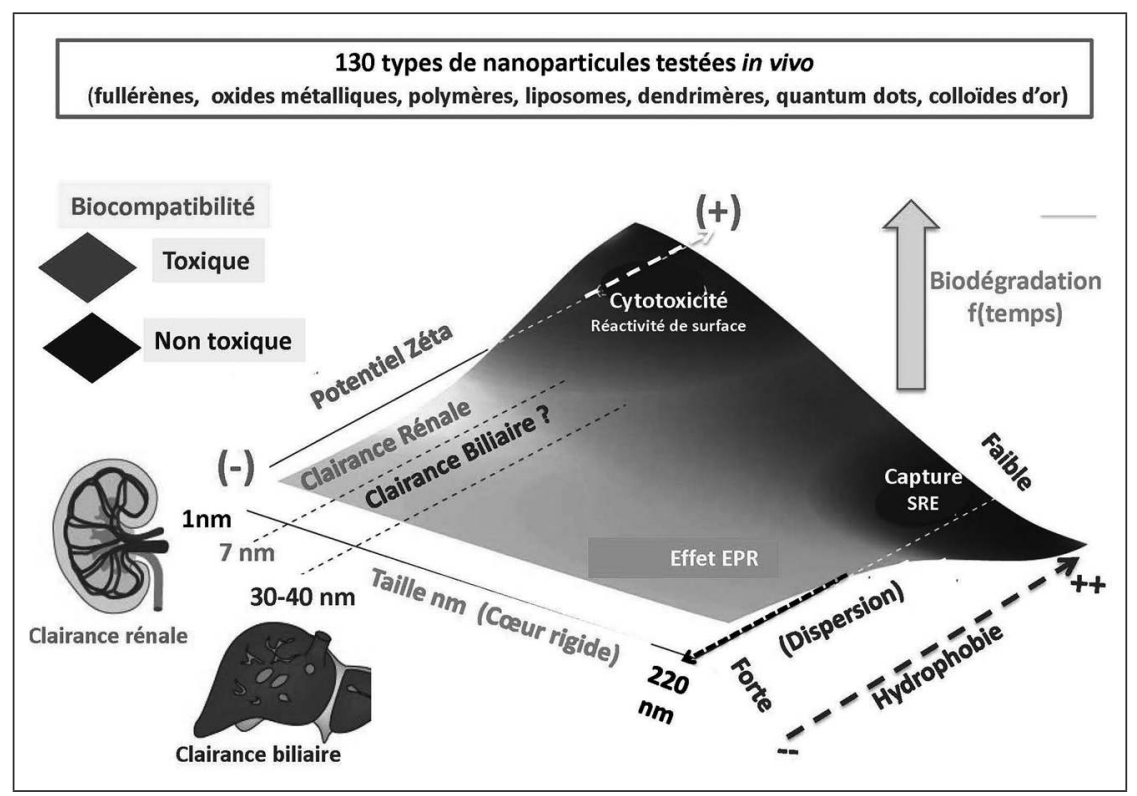

Figure 1 : Biocompatibilité in vivo des nanoparticules ; adapté d'après McNeil S.E., « Nanoparticle therapeutics », Nanomed. Nanobiotechnol., 1, 2009, 264-271.

médecine. Les principales variables indépendantes qui déterminent la biocompatibilité in vivo des nanoparticules (spectre de couleurs) sont la taille, le potentiel zêta (charge de surface) et la dispersibilité (en particulier l'effet hydrophobe). Le diagramme met clairement en évidence des zones dans lesquelles on attend un comportement toxique ou biocompatible des NP. L'utilisation optimale de nanoparticules dans des applications biomédicales nécessite par conséquent un parfait contrôle non seulement de leur propriétés chimiques intrinsèques (composition, structure), mais aussi de leur surface. Lorsque l'on souhaite atteindre une cible à des fins thérapeutiques, il faut astucieusement camoufler les nano-vecteurs afin qu'ils ne soient pas trop vite reconnus et donc éliminés par le système réticulo-endothélial. Une leçon a été entièrement consacrée à l'étude des différents et principaux modes de camouflages utilisés. La petite taille des NP de silice associée à la pégylation de leur surface, le contrôle de la charge des NP et de la stabilité des dispersions même après formation de la couronne protéique, le masquage de certaines fonctionnalités, la présence d'un nombre contrôlé de molécules capables de cibler les biocomposantes surexprimées par des cellules cancéreuses font partie des principales stratégies de camouflage et de ciblage utilisées. Plus récemment, l'utilisation préalable des phénomènes d'endocytose et d'exocytose de NPS pour construire de nouveaux vecteurs biogéniques ouvre de nouvelles perspectives. Ces compartiments biohybrides microvésiculaires qui permettent d'échanger, de vectoriser des principes actifs ou des sondes de fonctionnalités variées devraient ouvrir des perspectives intéressantes en nanomédecine. 


\section{Oxydes métalliques et stress oxydant}

La production de nanomatériaux manufacturés représente une percée scientifique et technologique indiscutable. Cependant, bien que les nanotechnologies puissent être à la fois des solutions innovantes aux grands défis sociétaux (énergie, santé, environnement) et un vecteur permettant la croissance de l'économie mondiale, nous devons également tenir compte de l'impact des propriétés physico-chimiques des nanoparticules sur l'environnement et sur les systèmes biologiques. Afin d'évaluer les dangers des nanoparticules de manière fiable et reproductible, des approches de dépistage sont nécessaires. En particulier, de nombreux laboratoires de recherche commencent à développer des plateformes de test afin d'analyser les interactions bio-physico-chimiques à l'interface nanomatériaux / biologie. L'étude des effets et mécanismes mis en jeu permet une approche toxicologique prédictive. L'utilisation de méthodes de criblage à haut débit (CHD) in vitro couplées à de l'analyse statistique s'appuyant sur des librairies combinatoires permet de prédire quels sont les nanomatériaux qui peuvent conduire à la génération de pathologies ou de maladies. Les résultats des tests in vivo sur animal et sur cellules humaines ou lignées cellulaires implantées sont utilisés pour valider et améliorer le système de tests in vitro par MCHD. La modélisation associée à une combinaison appropriée des résultats in vitro et in vivo permet d'établir de manière robuste les relations structure-activité des nanomatériaux et de les classer en fonction des risques de toxicité. Dans ce cadre, nous avons centré notre propos sur l' analyse nanotoxicologique de particules à base d'oxydes métalliques de formule $\mathrm{MxOy}(\mathrm{M}=\mathrm{Al}, \mathrm{Ti}, \mathrm{Co}, \mathrm{Cr}$, $\mathrm{Ce}, \mathrm{Fe}, \mathrm{Gd}, \mathrm{Hf}, \mathrm{La}, \mathrm{Mn}, \mathrm{Ni}, \mathrm{Sb}, \mathrm{Sn}, \mathrm{Si}, \mathrm{W}, \mathrm{Y}, \mathrm{Zn}, \mathrm{Yb} \ldots$... En particulier, dans ce cas, le stress oxydant créé par certains oxydes et / ou la solubilité du nano-objet sont les principales causes de toxicité. Le stress oxydant peut en particulier générer des pathologies diverses (vieillissement, cancer, inflammations chroniques, maladies neurodégénératives). Il y a trois niveaux de réponse cellulaire au stress oxydant par rapport auxquels l'activité des différentes nanoparticules a pu être classé : la défense anti-oxydant, l'inflammation, la cytoxicité. L'analyse des données publiées permet d'établir un paradigme prédictif en toxicologie et l'évaluation des risques présentés par les nanomatériaux de cette famille d'oxydes. Pour le stress oxydant en particulier, il semblerait qu'il existe une bonne corrélation entre la toxicité du nano-objet et la position énergétique de sa bande de conduction par rapport au potentiel d'oxydoréduction du couple $\mathrm{H}+/ \mathrm{H}_{2}$. Dans le cadre de cet ensemble d'analyses, les nanoparticules des oxydes de nickel, cobalt, manganèse et chrome s'avèrent nettement toxiques car elles génèrent un stress oxydant important. Par contre, la cause principale de la toxicité des nanoparticules d'oxydes de zinc et de cuivre est associée à leur forte solubilité qui entraîne des ruptures lysosomales par effet de pompe à protons. La cause étant connue, la toxicité du $\mathrm{ZnO}$ peut être très fortement diminuée par insertion de fer dans la structure, ce dernier diminuant fortement la solubilité du nano-objet. En effet, l'ensemble des tests in vitro (CHD) et in vivo (sur souris et poisson-zèbre) semblent montrer une absence de toxicité pour des taux de substitution de $8 \%$ du zinc par le fer. Les autres oxydes étudiés ne répondant pas positivement à l'ensemble des tests de toxicité in vitro (CHD) et in vivo sont peu ou ne sont pas toxiques. Certains d'entre eux sont utilisés comme agents de contraste dans l'imagerie par résonance magnétique nucléaire (oxyde de fer magnétique), en médecine régénérative pour soigner la dégénérescence maculaire (oxyde de cérium), comme sonde luminescente (oxydes de lanthanides) ou comme nanovecteurs 
thérapeutiques hybrides pour la thérasnostique (silice). Ce dernier matériau, la silice, est au centre de nombreuses recherches et de développements industriels (en cosmétique et nanomédecine). Cependant, l'analyse de la littérature concernant la toxicité des silices semblait montrer une disparité dans les conclusions des études menées par différents auteurs. C'est pourquoi, au cours de la leçon suivante, nous avons fait le point sur la toxicité de la silice.

\section{$\mathrm{SiO}_{2}$ en milieu biologique : faisons un point}

Le dioxyde de silicium $\mathrm{SiO}_{2}$ est le minéral le plus abondant sur terre. Il existe principalement sous forme de cristaux (quartz, cristobalite), de verres et de silices artificielles amorphes. Les applications des oxydes de silicium sont très nombreuses et couvrent cinq principaux domaines industriels : l'électronique et la bijouterie (résonateurs, oscillateurs, capteurs, pierres semi-précieuses), la cosmétique et l'alimentation (relargage d'huiles et de parfums, antibactériens, dentifrices, protection solaire...), la chimie de spécialité (ajouts pour les matériaux de construction, encapsulation et relargage de composés, charges minérales dans les polymères et dans les pneus...), la santé (délivrance de principes actifs peu solubles, composants de pansements et de cicatrisants). Dans le cadre de leurs nombreuses applications, les dioxydes de silicium peuvent être élaborés ou utilisés sous forme de poudres dont les grains sont de taille micronique, submicronique voire nanométrique. Ces formes pulvérulentes peuvent être également générées par abrasion au cours de la réalisation de certains métiers (construction et isolation, travaux dans les carrières, mineurs, porcelainiers, prothésistes...). Par conséquent, il nous a semblé important de faire le point sur les connaissances actuelles concernant la toxicité des dioxydes de silicium $\mathrm{SiO}_{2}$. Les poudres de silice cristalline (quartz, cristobalite) et celles issues des verres industriels entraînent des inflammations des voies respiratoires (fibrose, œdème, cancer du poumon) et sont cytotoxiques. Cette cytotoxicité est due au fait que le broyage de ces silices cristallines peut créer des concentrations très élevées de radicaux en surface (ROS pour Radical Oxygen Species) qui génèrent un fort stress oxydant. De plus, cette toxicité peut être accrue car certains quartz ou cristobalites peuvent être contaminés par du fer dont la présence entraîne des réactions de type Fenton, source supplémentaire de formation de radicaux libres hydroxyles. Le diagnostic est beaucoup moins évident en ce qui concerne les silices amorphes, souvent utilisées sous forme de poudres nanostructurées dans des applications comme les charges minérales pour améliorer le contrôle rhéologique et les comportements mécaniques, les catalyseurs, les desséchants, les dentifrices, les vecteurs cosmétiques ou thérapeutiques. Ces silices amorphes sont produites en grandes quantités (la production mondiale en nanoparticules de silice amorphe a été estimée à 1,3 tonne par an en 2000), et elles représentent de ce fait les nanoparticules synthétiques les plus abondantes sur terre.

Les nanoparticules de silice amorphe sont préparées suivant deux modes de synthèse principaux : à haute température $\left(1200\right.$ à $\left.1400{ }^{\circ} \mathrm{C}\right)$ par pyrolyse à la flamme de précurseurs chlorés $\left(\mathrm{SiCl}_{4}\right)$ suivie d'une trempe thermique rapide pour former ce que l'on appelle des «fumées de silice », ou par chimie douce en solution aqueuse via des réactions de polycondensation mettant en jeu des groupes silanols ( $\equiv \mathrm{Si}-\mathrm{OH}+\mathrm{HO}-\mathrm{Si} \equiv \Leftrightarrow \equiv \mathrm{Si}-\mathrm{O}-\mathrm{Si} \equiv$ ) pour former des silices colloïdales denses ou mésoporeuses. 
Les silices amorphes manquent d'ordre à grande distance et, en raison d'un paysage énergétique plat, leurs structures sont fortement dépendantes de facteurs cinétiques et environnementaux. Ces facteurs se manifestent principalement par des différences dans: (i) l'architecture du réseau siloxane, qui consiste en une combinaison de cycles, parmi lesquels la concentration, le motif (nombre de chaînons constituant le cycle) et la répartition (dans l'ensemble du matériau ou principalement près de la surface), (ii) l'étendue du réseau de liaisons hydrogène formé par les groupements silanols ( $\equiv \mathrm{Si}-\mathrm{OH})$.

Nous avons discuté des relations structure / toxicité pour des nanoparticules de silice amorphe de même taille synthétisées à basse température ou à haute température. Sur la base de tests hémolytiques sur érythrocytes, de l'évaluation de la viabilité cellulaire et des niveaux d'ATP dans des cellules épithéliales et des macrophages, il apparaît nettement que les fumées de silice présentent une toxicité importante, qu'elles soient fraîchement préparées ou recuites ou même après vieillissement à l'air. En revanche, les silices colloïdales, élaborées par chimie douce, dans des conditions de traitement identique, sont non toxiques. En particulier, pour les fumées de silice, il existe une bonne corrélation entre la toxicité et la concentration en groupements hydroxyles et leur potentiel à générer des espèces réactives de l'oxygène (ROS) qui provoquent l'hémolyse des globules rouges. Grâce à la combinaison de caractérisations spectroscopiques (spectrométries vibrationnelles Raman et infra-rouge, résonance paramagnétique électronique...) et d'analyses physiques (diffusion de rayons $\mathrm{X}$, microscopies), la détermination de l'état d'agrégation des silices, de la concentration en groupements hydroxyles, de la proportion relative de cycles de siloxane contraints et non contraints et leur potentiel à générer des radicaux hydroxyles, l'origine structurale de la toxicité de certaines silices amorphes a pu être mieux cernée. La toxicité des fumées de silice semble principalement provenir de leur importante population de cycles contraints à trois chaînons créés par leur mode de synthèse et de leur forte concentration en groupements hydroxyles. Les liaisons hydrogène et les interactions électrostatiques des silanols à la surface des agrégats des fumées de silice entraînent de fortes perturbations de la membrane plasmique extracellulaire détectées par des inflammasomes dont l'activation ultérieure conduit à la sécrétion de cytokines.

Les radicaux hydroxyles engendrés par les petits cycles tendus fortement présents dans les fumées de silice mais très minoritaires dans les silices colloïdales contribuent fortement à l'activation de l'inflammasome.

En conclusion, il semble que la cristallinité ne soit pas une condition préalable de la toxicité des oxydes de silicium. Certaines nanoparticules de silice amorphe présentent des toxicités au moins égales voire supérieures à celles des silices cristallines. Dans les silices amorphes, la production de ROS est fortement promue par la présence de cycles siloxanes tendus à trois chaînons formés à haute température et fortement instables à l'hydrolyse. Ces cycles tendus sont facilement observables par spectroscopie Raman et sont absents dans toutes les silices colloïdales (silice Stöber, mésoporeux de silice, silices LUDOX, gels de silicalite et gels de silice) qui, sur la base des tests effectués dans la littérature, ne présentent pas de toxicité. Ces petits cycles présents dans les fumées de silice synthétisées à haute température et dans la silice vitreuse servent de réservoir de ROS qui peuvent être générés continûment via l'hydrolyse de la surface ou la dissolution des nanoparticules, ce qui explique en grande partie la toxicité observée pour ces silices. L'utilisation de revêtements polymériques ou de bicouches lipidiques masque les interactions entre 
les cellules et les groupements hydroxyles et, par conséquent, diminue voire supprime la cytotoxicité. La stratégie qui est utilisée dans les travaux récents concernant les vecteurs de principes actifs est d'une part de sélectionner des silices amorphes élaborées par chimie douce et, d'autre part, de les recouvrir par une couche organique hybridante porteuse de multiples fonctionnalités. Cette double condition rend plus fiable l'utilisation de ces nanomatériaux.

\section{Leçons consacrées aux diatomées. Diatomées : belles et utiles diatomées, de la dynamite au cristal photonique}

Les diatomées sont des micro-algues photosynthétiques unicellulaires vivant aussi bien dans l'eau douce que dans l'eau de mer. Il en existe plus de 250 genres et plus de 200000 espèces. Les diatomées possèdent une coque appelée « frustule », constituée de silice amorphe. Ces frustules présentent des architectures 3D complexes obtenues par auto-assemblage génétiquement contrôlé d'éléments nanométriques régulièrement disposés (pores, canaux, protubérances) (figure 2). Ces structures très esthétiques et fonctionnelles ont inspiré aussi bien les artistes du nano-art que les architectes et les ingénieurs lors de la conception de bâtiments et d'aéronefs. Utilisant la photosynthèse comme source d'énergie (conversion photoassistée du dioxyde de carbone et de l'eau en sucres et oxygène), les diatomées vivent là où la lumière du soleil peut pénétrer, c'est-à-dire dans la zone euphotique située entre la surface de la mer ou du lac et des profondeurs de l'ordre de $200 \mathrm{~m}$. Elles sont essentielles à la vie sur Terre, puisqu'elles produisent environ $20 \%$ de

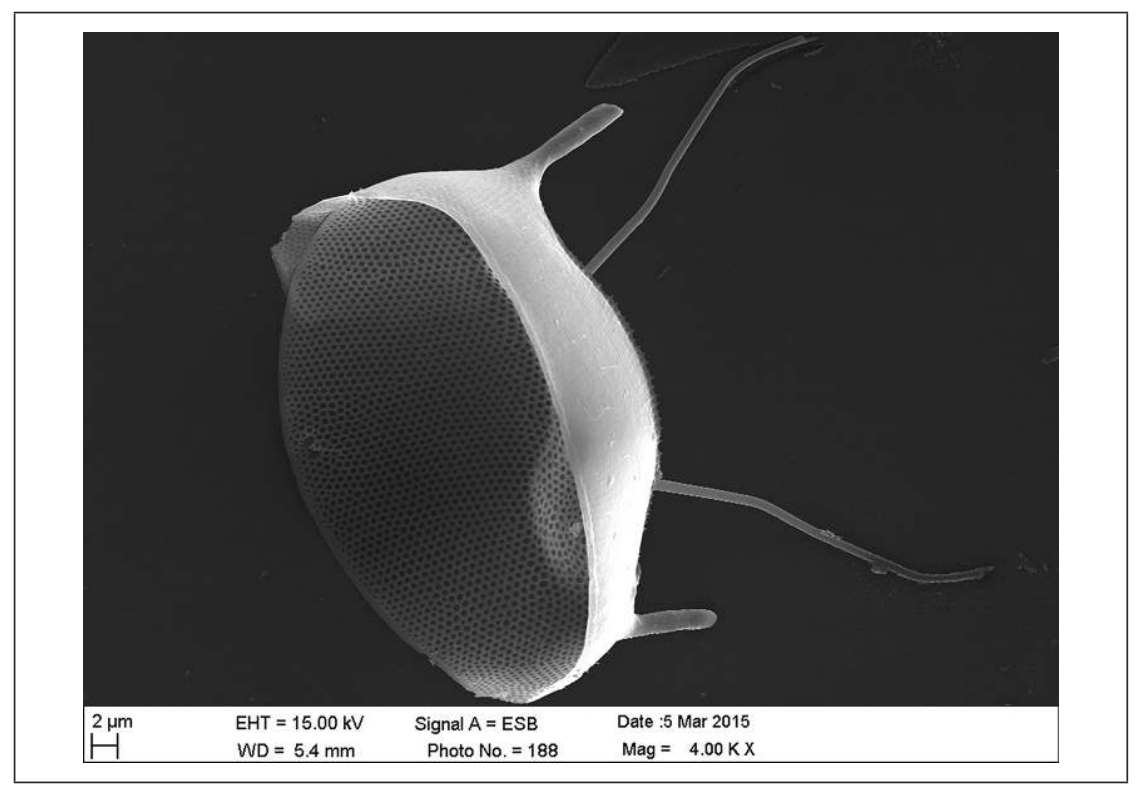

Figure 2 : Frustule en silice de diatomées.

(C) Laboratoire de Chimies de la matière condensée, Collège de France, UMR 7574 
l'oxygène que nous respirons. Lorsqu'elles meurent, elles sédimentent au fond de l'océan, produisant ainsi un immense puits à dioxyde de carbone. Leur rôle est donc essentiel dans le cycle du carbone de la Terre.

Ces micro-algues sont également de riches modèles pour l'étude des processus de biominéralisation de la silice. Le fait que les génomes de plusieurs diatomées aient été séquencés dans la dernière décennie, associé aux progrès réalisés dans les méthodes physico-chimiques de caractérisation, a permis de clarifier en partie les relations entre les biomacromolécules présentes, les processus d'auto-assemblage et la construction des architectures complexes en silice. Trois groupes de biomolécules sont fortement associées au processus de biosilification : les silaffines, les silacidines et les polyamines à longue chaîne. Les silaffines et silacidines sont des peptides ou des protéines qui portent de nombreux résidus phosphate attachés à des acides aminés (sérine et thréonine), alors que les polyamines à longue chaîne sont des composantes non protéiques essentiellement linéaires constituées de chaînes oligopropylèneimine. Via des processus d'auto-assemblage, essentiellement sous contrôle électrostatique, ces macromolécules biologiques forment des bio-agrégats. Au premier ordre, la taille des bio-agrégats et la quantité d'oligomères siliciques qui s'y lient déterminent la taille finale des sphères de silice dans l'architecture silicique de la diatomée. In vitro, ces biomolécules ont aussi la capacité d'influencer considérablement la cinétique de formation et la structure des silices obtenues. La compréhension de ces mécanismes est une source d'inspiration pour développer de nouveaux procédés de synthèse et des structures hybrides organique-inorganique originales. Du point de vue de la recherche fondamentale, les diatomées sont susceptibles de contribuer à la résolution de l'une des questions encore non totalement résolues en biologie : quelle est l'implication précise du génome dans la création des formes et quels sont les leviers de contrôle de leurs évolutions ? Bien que, pour un petit ensemble de diatomées, quelques hypothèses concurrentes aient été émises récemment, il faut se garder de conclusions hâtives. Il est nécessaire d'amplifier les observations et les analyses physico-chimiques intracellulaires pour mieux comprendre la nature et la dynamique des phénomènes en jeu.

En termes de science des matériaux, les diatomées permettent de développer de nouvelles voies de recherche et de développement. Les architectures fortement poreuses de leurs frustules ont notamment permis d'utiliser les diatomites (diatomées fossiles) comme adsorbeurs de molécules toxiques et de métaux lourds, supports de molécules réactives et de catalyseurs, filtres et membranes, vecteurs de principes actifs en agriculture et en cosmétique. En raison de leur abondance et de l'évolution structurale de leurs frustules en fonction de la pollution, les diatomées sont aussi utilisées comme indicateurs de la qualité de l'eau. Les diatomées pouvant être produites par aquaculture et présentant une grande diversité dans leurs nanostructures, micro-structures et morphologies, elles sont une source facilement accessible et suffisamment abondante pour permettre l'obtention de quantités nécessaires à la réalisation d'études en science des matériaux.

Plus particulièrement, au cours des dix dernières années, depuis l'explosion des nanotechnologies, de nombreux travaux de recherches essayent de tirer partie des structures poreuses multi-échelles des diatomées. Les applications potentielles de ces nouveaux matériaux ou dispositifs à base de diatomées ont été abondamment présentées et discutées dans le cadre de la sixième leçon. La modification chimique et/ou biochimique des frustules des diatomées conduit à l'obtention de matériaux présentant un ensemble de propriétés originales. Les frustules en silice poreuse 
acceptent des taux de principes actifs élevés, sont biocompatibles et facilement fonctionnalisés par des composantes organiques ou biologiques. Ce qui permet d'élaborer des vecteurs thérapeutiques hybrides pour la délivrance contrôlée de médicaments. Des vecteurs pour la théranostique couplant ciblage, diagnostique et thérapie ont déjà été réalisés. Des études récentes ont également démontré que ces micro-algues peuvent être une source durable de biocarburants. Les structures des nanopores des frustules sont semblables à celles des cristaux photoniques dans lesquels les photons se propagent comme les électrons dans un cristal semiconducteur, générant des zones d'énergie permise ou interdite. Grâce à cette propriété, les frustules peuvent servir de micro-lentilles pour optimiser les réponses optiques et concentrer la lumière dans des dispositifs de conversion et de stockage de l'énergie tels que des cellules photovoltaïques, cellules solaires à colorant, cellules photoélectrochimiques, batteries photo-rechargeables... D'autre part, la transformation topotactique des diatomées en différents matériaux (semiconducteurs, conducteurs, piézoélectriques: $\mathrm{TiO}_{2}, \mathrm{BaTiO}_{3}, \mathrm{Si}$, nanocomposites diatoméegraphène, etc.) ouvre de nombreuses possibilités pour l'élaboration de matériaux dont les propriétés irriguent les domaines de l'énergie et de l'environnement. En particulier, les propriétés de photoluminescence ou de conduction de ces matériaux sont sensibles à l'adsorption de gaz, de molécules ou de biomolécules. Cette sensibilité amplifiée par la porosité multi-échelle des frustules permet l'élaboration de capteurs à gaz et de biocapteurs performants. La transformation des frustules de silice en l'élément silicium permet d'élaborer des batteries Li-ion à électrode négative en silicium dont les performances sont prometteuses.

Les diatomées peuvent également former des suspensions colloïdales stables permettant l'obtention par lithographie de composants de circuits en microélectronique ou de réseaux de capteurs. Ces composantes sont modifiables chimiquement dans une seconde étape par ALD (Atomic Layer Deposition) ce qui permet encore de diversifier les fonctionnalités accessibles.

Sans aucun doute, une meilleure compréhension de la structure des diatomées et de leur génomique, de leurs propriétés mécaniques, optiques et photoniques et de leur processus de bio-minéralisation conduiront à la nano-fabrication et à l'ingénierie de nouveaux matériaux et dispositifs. Les diatomées continueront à jouer un rôle important en biologie et en science des matériaux car leur beauté ne cesse d'inspirer tous ceux qui les étudient.

\section{Séminaire. Interfaces : chimie des matériaux, biologie, médecine ${ }^{b}$}

Le séminaire a pris la forme d'un colloque qui s'est tenu au Collège de France le 17 mars 2015. Deux conférences plénières et treize conférences invitées ont été présentées. Les conférences plénières présentées par le professeur Peter Fratzl (Institut Max Planck des Colloïdes et interfaces, Potsdam, Allemagne) et le professeur Juan Manuel Garcia-Ruiz (CSIC, université de Grenade, Espagne) portaient respectivement sur de nouveaux résultats concernant la croissance des biominéraux et la capacité de la silice à mimer la matière bio-organique dans des structures minérales abiotiques de type «biomorphes » obtenues par auto-assemblage minéral-minéral. Les titres des

b. Le colloque est disponible en vidéo sur le site internet du Collège de France : http:// www.college-de-france.fr/site/clement-sanchez/symposium-2014-2015.htm [NdÉ] 
conférences invitées présentées et les orateurs sont les suivants : "Vésicules polymères biomimétiques et nanomédecine », par le Pr Sébastien Lecommandoux (université de Bordeaux), « Cycle de vie et biodégradation de nanoparticules dans l'organisme », par la Pr Florence Gazeau (université de Paris VII), "Cristaux liquides biologiques et médecine réparatrice, quel avenir?», par la Pr Marie-Madeleine Giraud-Guille (EPHE), «L'importance de l'eau aux interfaces hybrides, une étude par RMN du solide », par le Dr Thierry Azais (université de Paris VI), « Nouveaux outils pour la mise en forme du collagène et Ies implications dans le domaine biomédical », par le Dr Francisco Fernandes (université de Paris VI), «Du concept d'un matériau à la différentiation cellulaire: une approche transversale», par le Pr Jean Le Bideau (université de Nantes), «Luminescence persistante confinée dans une nanoparticule : futurs traceurs pour l'imagerie in vivo ? » par la Pr Corinne Chanéac (université de Paris VI), "Systèmes magnétiques hybrides : du diagnostic à la thérapie », par la Pr Christine Ménager (université de Paris VI), « Morphogenèse de capsules cœurécorces fonctionnelles par chimie intégrative: délivrance thermostimulable et confinement bactériologique », par le Pr Rénal Backov (université de Bordeaux), «Associer les molécules biologiques aux particules inorganiques pour élaborer des biomatériaux fonctionnels et modulables », par la Dr Carole Aimé (CNRS / université de Paris VI), «Nouvelles stratégies d'élaboration de vecteurs par le Dr Cédric Boissière » (CNRS / université de Paris VI), «Silices hybrides multifonctionnelles : applications potentielles en nanomédecine », par le Dr Michel WCM (CNRS / université de Montpellier II), «De la lithiase aux calcifications pathologiques : une recherche à l'interface physique, chimie et médecine », par le Pr Michel Daudon (CHU Tenon, Paris), "Matériaux combinés pour le traitement de pathologies osseuses », par le Pr Bruno Bujoli (université de Nantes).

\section{RECHERCHE}

L'équipe, intitulée «Matériaux hybrides et nanomatériaux », dirigée et animée par Clément Sanchez, est l'une des trois équipes du laboratoire de Chimie de la matière condensée de Paris (UMR UPMC / Collège de France / CNRS no 7574). Les travaux de l'équipe se déroulent le long de quatre «axes de recherches concertées » (ARC) principaux dont les activités concernent la synthèse de nanomatériaux inorganiques, les nanomatériaux pour l'énergie, l'élaboration de matériaux hybrides à composante polymère, l'étude des couplages chimie-procédés, et dont les animateurs sont respectivement : C. Chanéac, C. Laberty, L. Rozes et C. Boissière. La description exhaustive de toutes les activités de recherche et résultats de l'équipe et de ses quatre ARC n'étant pas le but de ce rapport, nous nous limiterons à décrire quelques faits marquants de la période juin 2014 juin 2015 obtenus dans chacun des ARC. Les collègues cités dans l'ARC sont ceux ayant participé aux travaux illustrés. La liste de l'ensemble de nos publications communes est ensuite présentée à la fin de la partie recherche (vide infra).

\section{Nanomatériaux inorganiques}

\section{Goldman, D. Portehault, C. Chanéac, C. Sanchez}

Nous allons illustrer notre activité dans cet ARC via nos approches moléculaires pour la synthèse de nanomatériaux exotiques. En effet, les synthèses par réaction 
entre des précurseurs moléculaires sont souvent les plus adaptées pour la fabrication et la mise en forme à faible coût de nouveaux matériaux à structure, nanostructure et porosité contrôlées. La fonctionnalisation de surface de colloïdes est indispensable à leur stabilité et à leur fonction d'usage, que ce soit pour des applications en biomédecine, en catalyse ou pour la formulation de produits manufacturés. Néanmoins, elle peut fortement impacter les propriétés intrinsèques des colloïdes. À partir d'un système modèle de colloïdes d'or, nous nous sommes intéressés à des effets de compositions chimiques de la couche fonctionnelle et d'hétérogénéités de surface sur les propriétés optiques de l'or nanométrique.

De très nombreux travaux se sont focalisés sur l'influence de la fonction d'ancrage sur les surfaces d'or (thiol, phosphine, acétate, etc.) mais, paradoxalement, aucune étude n'a été menée sur l'influence de la chaîne latérale portée par une même fonction d'ancrage. Nous avons ainsi modulé la polarité et la nature des interactions intermoléculaires de ligands thiols en utilisant des chaînes alkyles, des unités éthylène glycol ou des cycles aromatiques. Un premier résultat marquant a été observé en présence de ligands aromatiques (possédant de 1 à 3 cycles conjugués) qui induisent un déplacement de la bande plasmon vers les grandes longueurs d'onde d'autant plus important que le nombre de cycles aromatiques est élevé, et qui s'accompagne d'une augmentation de l'absorbance des suspensions. Par l'intermédiaire de techniques spectroscopiques avancées et de modèles théoriques, l'origine du déplacement de la bande plasmon en fonction de la nature du ligand et du nombre de cycles aromatiques a été attribuée à une intensification des transitions interbandes dans le cœur d'or [1].

Afin de générer de l'hétérogénéité de surface, des échanges de ligands thiols ont été réalisés et suivis en temps réel par RMN. Cette approche originale a notamment montré des différences structurales importantes qui peuvent se traduire par des capacités d'échange très variées. Globalement, les ligands qui possèdent des chaînes polyéthylène glycol déplacent les alkylthiols bien plus difficilement que les ligands aromatiques du fait d'interactions intermoléculaires moins favorables à la stabilité de la monocouche. Nous avons également mis en évidence que l'efficacité des échanges dépendait de la courbure de la surface, elle-même influant sur les interactions intermoléculaires. Si cet effet est intuitif, sa preuve expérimental est un résultat considérable. Par ailleurs, la ségrégation des ligands dans ces systèmes a été étudiée par RMN NOESY et comparée à des synthèses de nanoparticules d'or bi-ligands. Pour des nanoparticules de $5 \mathrm{~nm}$, les ligands alkylthiol et aromatiques sont proches spatialement alors que les ligands alkylthiol et thiol dibloc alkyleéthylène glycol sont fortement ségrégés dans la monocouche. Ces études sont d'autant plus complexes à réaliser qu'il est nécessaire de s'assurer à tout moment que ces cinétiques d'échanges n'engendrent pas de restructuration du cœur de la nanoparticule [2].

La ségrégation des ligands pour des colloïdes en milieu aqueux est plus délicate à mettre en œuvre et a nécessité de développer une autre méthodologie utilisant la tomographie électronique. Différents couples de ligands ont été étudiés, mettant à chaque fois en jeu un ligand dit «réactif» terminé par une fonction acide carboxylique (de longueur de chaîne variable) et un ligand « inerte » chimiquement terminé par une chaîne polyéthylène glycol. L'ajout d'un précurseur de silice en milieu basique a permis de condenser de la silice sur les zones enrichies en ligand réactif alors observables en tomographie. La morphologie de la silice, en couches concentriques, asymétriques ou sous forme de lobes, traduit la ségrégation des 
ligands. Les ligands possédant un bloc alkyle et une fonction amide forment des domaines ségrégés lorsqu'ils sont couplés à un acide à longue chaîne (acide C16). Cette ségrégation a probablement pour origine les interactions intermoléculaires de type liaisons hydrogène (via la fonction amide) et interactions de Van Der Waals (via le bloc alkyle) présentes dans ces composés. À l'inverse, la seule présence d'une chaîne polyéthylène glycol ne permet de développer que très peu d'interactions intermoléculaires favorables à l'auto-assemblage et ne produit pas de ségrégation dans la couronne de ligands. Actuellement, nous orientons cette thématique de recherche sur des nanoparticules d'or et des nanoclusters d'or stabilisés par des carbènes boranes dont les récentes publications sur SAMS suggèrent des effets importants sur les structures électroniques de l'or.

[1] C. Goldmann, R. Lazzari, X. Paquez, C. Boissiere, F. Ribot, C. Sanchez, C. Chaneac, D. Portehault, «Charge Transfer at Hybrid Interfaces: Plasmonics of Aromatic Thiol-Capped Gold Nanoparticles», ACS Nano, 9(7), 7572-7582 (2015)

[2] C. Goldmann, F. Ribot, F. Tielens, C. Sanchez, C. Chaneac, D. Portehault, « Building a Surface Binding Scale by Quantification of Ligands Exchange onto Gold Nanoparticles: the Role of Curvature and Intermolecular Forces», soumis à ACS Nano.

\section{Nanomatériaux et énergie}

\section{N. Krins, E. Courtin, C. Laberty, C. Sanchez}

Cet axe de recherche s'organise autour de trois thèmes principaux : les matériaux hybrides pour le transport d'espèces chargées, les matériaux inorganiques pour la conversion et le stockage de l'énergie et l'élaboration de composites hybrides contenant des organismes du vivant pour la conversion de l'énergie. C'est plus particulièrement les activités associées au thème des batteries photorechargeables que nous illustrerons cette année.

La technologie des accumulateurs (ou batteries rechargeables) Li-ion classique repose sur le stockage réversible de l'énergie électrique en énergie chimique. Cette dernière résulte de la différence de potentiel électrochimique qui existe entre un certain matériau, riche en ions lithium (électrode négative) et un autre, pauvre en ions lithium (électrode positive). Quand vous utilisez votre téléphone, on dit qu'il se décharge, ce qui signifie que les ions lithium, initialement présents dans l'électrode négative, se déplacent, via un électrolyte, pour rejoindre le matériau d'électrode positive et s'y insérer. Recharger votre téléphone consiste à imposer aux ions lithium le chemin inverse. Mais cette opération ne sera possible que si, en échange, vous donnez de l'énergie à votre système, ce que vous faites en le connectant au secteur : vous lui fournissez de l'énergie électrique. Serait-il possible de le faire en utilisant une autre source d'énergie, une énergie renouvelable, par exemple? Dans nos travaux récents, nous avons montré que le soleil pouvait en effet remplir ce rôle. Nous avons donc introduit le concept de «batteries photorechargeables ». Le fait d'extraire, grâce à l'action de la lumière, des ions hors d'un matériau tel que l'oxyde de titane avait été introduit par un scientifique nommé Tribush dans les années 80. L'idée était lancée mais les rendements étaient extrêmement faibles, à cause essentiellement de l'utilisation de matériaux à l'échelle micronique. C'est seulement en 2014 que nous avons pu mettre au point des électrodes architecturées à base de nanomatériaux qui ont permis de photo-extraire en quantités les ions lithium présents dans le matériau d'électrode, conduisant ainsi 
à une recharge photo-induite du dispositif de batterie. Ceci a été possible grâce à notre connaissance de la chimie sol-gel et des procédés de mise en forme qui lui sont associés. D'un point de vue fondamental, ces recherches présentent un intérêt essentiel car elles permettent de revisiter le monde des batteries Li-ion à la lumière de l'expertise des réactions photo-induites comme celles rencontrées au sein des cellules photovoltaïques et / ou photoélectrochimiques. Dans le contexte global et urgent de la transition énergétique, beaucoup de chercheurs travaillent au développement de moyens capables de stocker l'énergie électrique provenant de la conversion efficace des énergies renouvelables. À l'heure actuelle, ces deux domaines de recherche - stockage et conversion - sont distincts et interagissent peu. Même si certains travaux montrent qu'il est possible de coupler ces deux types de dispositifs, la batterie photo-rechargeable constitue, à notre connaissance, le seul exemple qui combine, en un dispositif unique, les fonctions de stockage et de conversion. Bien nous ayons maintenant démontré le concept à l'aide d'électrodes de dioxyde de titane poreuses nanostructurées (une publication est en cours), les prochaines étapes vont être de comprendre finement les mécanismes physicochimiques mis en jeu afin de développer des batteries à longue durée de vie. Cette recherche à l'interface encore très peu explorée des batteries et des cellules photovoltaïques ouvre des perspectives intéressantes dans le domaine de la recherche à la fois fondamentale mais aussi appliquée.

1. Photorechargeable battery: turning light energy into chemical energy and then electric energy. French priority patent Application (2014) FR1361775.

2. E. Courtin, F. Sauvage, N. Krins, C. Sanchez, C. Laberty-Robert (en preparation), « $\mathrm{TiO} 2$ thin films as photoelectrodes for Li-ion insertion: towards the design of light rechargeable batteries », Journal Advanced Functional Materials.

\section{Matériaux hybrides à composantes polymères}

\section{F. Pottier, F. Ribot, L. Rozes, C. Sanchez}

Depuis plusieurs années, nos études portent sur la construction sur mesure de matériaux hybrides à composante polymère à partir de nano-objets calibrés (nanoparticules ou oxo-clusters métalliques). Cette année, parmi les recherches associées à cet axe, nous avons sélectionné une étude en cours concernant de nouveaux matériaux hybrides à mémoire de forme et présentant des propriétés d'autoréparation. Cette étude met en évidence la possibilité de concevoir des réseaux dynamiques basés sur la réticulation de matériaux polymères, élastomères ou thermoplastiques-élastomères, par des nanobriques inorganiques en générant une interface hybride ionique et donc labile.

Le cluster d'étain $\left[(\mathrm{BuSn})_{12} \mathrm{O}_{14} \mathrm{OH}_{6}\right] \mathrm{AMPS}_{2}$ a servi de nanobrique pour l'élaboration de matériaux autoréparants et thermoformables. Le cluster est composé d'un cœur oxo-métallique à 12 atomes d'étain, c'est un macrocation dont les charges sont compensées par deux anions acrylamidosulfonate. Grâce à ces ligands polymérisables, le cluster a été utilisé comme agent réticulant de chaînes de poly(acrylate de n-butyle). Le matériau hybride ainsi obtenu est un élastomère capable de récupérer l'ensemble de ses propriétés mécaniques après avoir subi un endommagement mécanique, allant de la fissure de quelques $\mu \mathrm{m}$ à un endommagement drastique (pièces monolithiques coupées en deux et réassemblées). En plus d'être réparable, le matériau est recyclable, ce qui est exceptionnel pour un élastomère. 
L'origine du comportement dynamique de l'élastomère hybride vient des liaisons ioniques entre le cœur du cluster et ses ligands qui connectent entre elles les chaînes macromoléculaires. Ces liaisons sont à la fois suffisamment fortes pour permettre de réticuler les chaînes et d'atteindre les propriétés d'un élastomère, et suffisamment labiles pour permettre au réseau d'être dynamique. Les propriétés mécaniques (essais de traction, tests de relaxation) des matériaux ont été évaluées et mettent en évidence la nature dynamique du réseau à l'échelle macroscopique. La RMN DOSY a permis quant à elle de mettre en évidence la dynamique dans le réseau à l'échelle moléculaire confirmant le rôle du cluster comme agent de réticulation à interfaces labiles.

En s'inspirant des nanostructurations hybrides à l'origine du phénomène de bioréparation des fibres des mollusques bivalves, de nouveaux copolymères à blocs ont été élaborés. Afin d'obtenir des matériaux avec des modules mécaniques élevés, l'incompatibilité entre les blocs a été mise à profit en synthétisant par polymérisation RAFT des réseaux hybrides nanoségrégés constitués de blocs rigides de polystyrène et de blocs souples de poly(acrylate de n-butyle) au sein duquel le cluster fonctionnel a été incorporé, assurant la réticulation dynamique du matériau. Les systèmes ainsi obtenus possèdent des propriétés de réparation thermostimulée, de thermoformage et peuvent être recyclés, contrairement aux élastomères thermoplastiques conventionnels. Cette stratégie de synthèse permet d'augmenter le module élastique de valeurs allant de $0,1 \mathrm{MPa}$ pour l'élastomère à $500 \mathrm{MPa}$ pour les copolymères, tout en conservant un caractère dynamique. Ces études démontrent qu'il est possible, via le design de matériaux hybrides de nano-objets-polymères, d'atteindre des propriétés d'autoréparation, de mémoire de forme, voire de thermoformage.

\section{Interface chimie-procédé}

\section{G. Drisko, C. Boissière, C. Sanchez}

Les activités de cet axe de recherche concernent l'élaboration de matériaux nanostructurés par combinaison de la chimie sol-gel avec des procédés de mise en forme ainsi que le développement de techniques de caractérisation in situ en temps résolu. La nanomédecine est un champ d'investigation multidisciplinaire dont l'ampleur n'a cessé d'augmenter dans la dernière décennie. Les avancées réalisées dans ce domaine impactent déjà fortement les méthodologies associées à la thérapie, au diagnostic, à l'imagerie de certaines maladies comme le cancer. En particulier, l'utilisation de nanoparticules astucieusement fonctionnalisées ouvre des perspectives immenses pour l'amélioration du diagnostic par imagerie magnétique ou optique, les traitements par hyperthermie, ainsi que l'adressage sélectif et la délivrance contrôlée de principes actifs. Cette année, nous avons élaboré et caractérisé des « hochets quantiques », des nanoparticules multifonctionnelles pour l'imagerie médicale multimodale et le traitement du cancer.

Les clusters d'or sont des nanoparticules possédant des propriétés quantiques (optiques et magnétiques) très différentes des nanoparticules d'or plus volumineuses. Toutefois, leur manque de stabilité en solution et leurs interactions défavorables avec les systèmes vivants en milieu aqueux ont jusqu'à aujourd'hui empêché leur emploi en biologie et médecine. Le travail récemment publié à PNAS, issu d'une étroite collaboration entre le Collège de France, l'Impérial College de Londres et l'University College de Londres, vient de montrer qu'il existe pourtant une méthode simple pour intégrer et stabiliser des clusters d'or au sein d'une matrice creuse de 
silice mésoporeuse, en coexistence avec des nanoparticules d'or plus grosses piégées dans la cavité centrale. Cette structure de « hochet quantique » est stable en solution aqueuse, très peu toxique, et préserve les propriétés photoniques (dans la gamme proche infra-rouge) et paramagnétique des clusters d'or, tout en permettant de maximiser leur capacité de stockage de médicament. Testés en expériences in vivo sur petit animal, les propriétés photo-thermiques hors norme de ces «hochets quantiques » ont permis de réduire significativement la masse de tumeurs en un seul traitement. En parallèle, les propriétés de ces vecteurs ont permis pour la première fois de coupler très simplement trois types d'imageries médicales complémentaires par fluorescence proche infra-rouge, imagerie photo-acoustique et IRM, permettant d'obtenir à la fois de très bonnes résolutions spatiales et temporelles d'analyses. L'incorporation d'or au sein de ces vecteurs a en outre permis d'accroître très significativement et de façon reproductible les capacités de stockage de doxorubucine, un agent anticancéreux souvent difficile à stabiliser dans ce genre de matrice poreuse. Le design innovant et la synthèse de ces vecteurs a permis de démontrer que l'usage de clusters d'or pour les applications couplées de diagnostic et de nanothérapie n'est plus un rêve mais bien une réalité qui va permettre de simplifier considérablement la composition de nano-vecteurs multifonctionnels, et donc d'envisager des temps de développement beaucoup plus courts pour les futurs nanomédicaments. Après optimisation, on peut également espérer une meilleure biodégradabilité et élimination de ces vecteurs car leurs composantes de silice, de clusters d'or et de principe actif ont des tailles et des solubilités aisément ajustables.

M. Hembury, C. Chiappini, S. Bertazzo, T.L. Kalber, G.L. Drisko, O. Ogunlade, S. WalkerSamuel, K.S. Krishna, C. Jumeaux, P. Beard, C.S.S.R. Kumar, A.E. Porter, M.F. Lythgoe, C. Boissière, C. Sanchez, M.M. Stevens, "Gold-silica quantum rattles for multimodal imaging and therapy », Proceedings of the National Academy of Sciences, 112, 1959 (2015).

\section{CONFÉRENCES ET SÉMINAIRES INVITÉS ${ }^{1}$}

«Integrative Chemistry based strategies to nanostructured inorganic and hybrid materials », Conférence à BASF, Ludwigshaffen, Allemagne (juillet 2014).

«Hybrid Organic-Inorganic Materials: basic chemistry, processing, applications », « Molecular Engineering of Mesoporous Materials », « Controlled design of hybrid materials built from functional nanobuilding blocks», International Summer School , Soft Fire, Cargese, Corse (juillet 2014).

«Integrative Chemistry: From Nanomaterials to Hierarchically Structured Inorganic and Hybrid Solids », International Conference on NanoMaterials for health, energy and environment, Mauritius (septembre 2014).

«Ingénierie moléculaire de nanomatériaux inorganiques et hybrides», École des Mines, CNRS, Lyon (septembre 2014).

«Integrative Chemistry based strategies to nanostructured inorganic and hybrid materials », E-MRS Varsovie (Pologne) (septembre 2014).

«Sol-gel derived nanostructured hybrid materials for the biomedical domain », colloque France-Japon, Drug Delivery Systems, Osaka, Japon (octobre 2014).

1. 30 juin 2014 - 30 juin 2015 . 
« Bioinspired and biomimetic approaches to advanced inorganic and hybrid materials », conference d'ouverture ED Matériaux de l'université de Séville (octobre 2014).

«Integrative Chemistry: From Nanomaterials to Hierarchically Structured Inorganic and Hybrid Solids », université de Séville (octobre 2014).

«Integrative chemistry based strategies to design nanostructured inorganic and hybrid materials », $4^{\text {th }}$ International Solvothermal Hydrothermal Association Conference: towards sustainability, Bordeaux, (octobre 2014).

«Integrative Chemistry to nanostructured inorganic and hybrid materials », HyMAp, International Conference on Hybrid Materials and Processing, Busan, Corée (novembre 2014).

«Ingénierie moléculaire de matériaux inorganiques et hybrides nanostructurés », ENS Cachan, Journée scientifique P. Audebert (octobre 2014).

« Molecular engineering of inorganic and hybrid nanomaterials », International Conference on Nanosciences and Environment, Dim Nano K, C'Nano, université de Paris 7 (novembre 2014).

«Vers des matériaux nanostructurés bioinspirés », Académie de Lorraine, Nancy (15 novembre 2014).

«Biomimétisme et bio-inspiration ?: sources d'innovation en science des matériaux », Matériaux 2014, Montpellier (novembre 2014).

«A short history of Hybrid Organic (Bio)-Inorganic Materials », IV International Conference on Hybrid Materials Sitges, Espagne (mars 2015).

«Chimie intégrative : une source d'innovation en science des matériaux », IFPEN, Lyon (20 mars 2015).

«Approches bio-inspirées : Comprendre pour créer en chimie des matériaux », Collège de France, journées Main à la pâte (23 mars 2015).

«Bottom up strategies for the construction of functional nanostructured inorganic and hybrid materials », XIX Congreso de Fisico-quimica y Quimica inorgnaica, Centro de convenciones Palais Rouge, Palermo, Buenos Aires (14 avril 2015).

«Applications of hybrid materials », «Nanomateriales orientados a la generación, conversión y almacenamiento de Energía », XIX Congreso de Fisico-quimica y Quimica inorgnaica, CNEA, San Martin, Buenos Aires (16 avril 2015).

«Biomimetismo y Bioinspiración: Una via de acceso a nuevos materiales híbridos e inorgánicos », Universidad de Granada, Espagne (12 mai 2015).

«Integrative chemistry: from nanostructured to hierarchically structured inorganic and hybrid materials », CSIC, Granada (13 mai 2015).

«Matériaux hybrides nanostructurés pour le domaine biomédical», colloque ERC Advanced Grant TERANOMED, Collège de France, mai 2015 (P. Couvreur, C. Sanchez).

«Integrative chemistry: The controlled design of inorganic and hybrid materials », rencontre L'Oréal-chaire de Chimie des matériaux hybrides, 27 mai 2015.

«Biomimetism and Bioinspiration: Sources of knowledge to create new materials», université de Trento, juin 2015.

«Integrative chemistry: from nanostructured to hierarchically structured inorganic and hybrid materials », université de Trento, juin 2015.

\section{ORGANISATION SCIENTIFIQUE DE COLLOQUES ET DE CONFÉRENCES}

Fourth International Conference on Multifunctional, Hybrid and Nanomaterials (Hybrid Materials 2015) Sitges, Espagne, 9-13 mars 2015. 
Colloque Collège de France, 17 mars 2015 :Interfaces : chimie des matériaux, biologie, médecine.

Colloque ERC Advanced Grant TERANOMED, P. Couvreur / Chimie des matériaux hybrides (C. Sanchez), Collège de France, 19 mai 2015.

Conférence de la Société chimique de France SCF'15, Chimie \& transition énergétique, Lille, juillet 2014.

\section{PuBLICATIONS $^{2}$}

\section{Articles}

Debecker D.P., Farin B., Gaigneaux E.M., SAnchez C. et SAssoye C., «Total oxidation of propane with a nano-RuO2/TiO2 catalyst », Applied Catalysis a-General, 481, 2014, 11-18, DOI : 10.1016/j.apcata.2014.04.043.

Depardieu M., Janot R., Sanchez C., Bentaleb A., Demir-Cakan R., Gervais C., Birot M., Morcrette M. et BACKOv R., « Novel Au/Pd@carbon macrocellular foams as electrodes for lithium-sulfur batteries », Journal of Materials Chemistry A, 2(42), 2014, 18047-18057, DOI : 10.1039/c4ta03537b.

Depardieu M., Janot R., Sanchez C., Bentaleb A., Gervais C., Birot M., DemirCAKAN R., BACKOV R. et MORCRETTE M., «Carbonaceous multiscale-cellular foams as novel electrodes for stable, efficient lithium-sulfur batteries », RSC Advances, 4(46), 2014, 23971-23976, DOI : 10.1039/c4ra03110e.

FERNANDES F.M., BARADARI H. et SANCHEZ C., «Integrative strategies to hybrid lamellar compounds: an integration challenge », Applied Clay Science, 100, 2014, 2-21, DOI : 10.1016/j.clay.2014.05.013.

Martinez E.D., Boissiere C., Grosso D., Sanchez C., Troiani H. et SolerILlia G.J.A.A., « Confinement-Induced Growth of Au Nanoparticles Entrapped in Mesoporous TiO2 Thin Films Evidenced by in Situ ThermoEllipsometry », Journal of Physical Chemistry C, 118(24), 2014,13137-13151, DOI : 10.1021/jp500429b.

Perineau F., Rosticher C., Rozes L., Chaneac C., Sanchez C., Constantin D., Dozov I., DAVIDSON P. et Rochas C., « Hybrid Nanocomposites with Tunable Alignment of the Magnetic Nanorod Filler », Acs Applied Materials \& Interfaces, 6(3), 2014, 1583-1588, DOI : 10.1021/am404313s.

Potier F., Guinault A., Delalande S., Sanchez C., Ribot F. et Rozes L., « Nanobuilding block based-hybrid organic-inorganic copolymers with self-healing properties », Polymer Chemistry, 5(15), 2014, 4474-4479, DOI : 10.1039/c4py00172a.

Hembury M., Chiappini C., Bertazzo S., Kalber T.L., Drisko G.L., Ogunlade O., Walker-Samuel S., Krishna K.S., Jumeaux C., Beard P., Kumar C.S.S.R., Porter A.E., Lythgoe M.F., Boissiere C., SAnChez C. et Stevens M.M., « Gold-silica quantum rattles for multimodal imaging and therapy », Proceedings of the National Academy of Sciences of the United States of America, 112(7), 2015, 1959-1964, DOI : 10.1073/pnas.1419622112.

Renault C., Nicole L., Sanchez C., Costentin C., Balland V. et Limoges B., «Unraveling the charge transfer/electron transport in mesoporous semiconductive $\mathrm{TiO} 2$ films by voltabsorptometry », Physical Chemistry Chemical Physics, 17(16), 2015, 10592-10607, DOI : $10.1039 / \mathrm{c} 5 \mathrm{cp} 00023 \mathrm{~h}$.

Drisko G.L., Carretero-Genevrier A., Perrot A., Gich M., GazQuez J., RodriguezCarvajal J., Favre L., Grosso D., Boissiere C. et Sanchez C., « Crystallization of hollow

2. 30 juin 2014 - 30 juin 2015. 
mesoporous silica nanoparticles », Chemical Communications, 51(20), 2015, 4164-4167, DOI : $10.1039 / \mathrm{c} 4 \mathrm{cc} 07828 \mathrm{~d}$.

Drisko G.L., Carretero-Genevrier A., Gich M., Gazquez J., Ferrah D., Grosso D., Boissiere C., Rodriguez-Carvajal J. et SAnchez C., «Water-Induced Phase Separation Forming Macrostructured Epitaxial Quartz Films on Silicon», Advanced Functional Materials, 24(35), 2014, 5494-5502, DOI : 10.1002/adfm.201401066.

Fernandez C., Sassoye C., Debecker D.P., SAnchez C. et Ruiz P., « Effect of the size and distribution of supported $\mathrm{Ru}$ nanoparticles on their activity in ammonia synthesis under mild reaction conditions », Applied Catalysis A-General, 474, 2014, 194-202, DOI : 10.1016/j. apcata.2013.09.039.

Barré C., Quet A., Bianchi L., SAnchez C. et SAuveroche A., « Chiral habit selection on nanostructured epitaxial quartz films, Spray plasma processed ZrB2-based coatings for oxidation protection », dans ITSC 2014 Conference proceedings, lectures and posters in Barcelona/Spain on May 21 - 23, 2014. International Thermal Spray Conference et exposition, Düsseldorf, DVS-Verlag, coll. « DVS-Berichte », no 302,2014, 541-545.

Carretero-Genevrier A., Drisko G.L., Grosso D., Boissière C. et Sanchez C., «Mesoscopically structured nanocrystalline metal oxide thin films », Nanoscale, 6(23), 2014, 14025-14043, DOI : 10.1039/c4nr02909g.

Debecker D.P., Boissière C., Laurent G., Huet S., Eliaers P., Sanchez C. et BACKOV R., "First acidic macro-mesocellular aluminosilicate monolithic foams "SiAl(HIPE)", and their catalytic properties », Chemical Communications, 51(74), 2015, 14018-14021, DOI : 10.1039/c5cc05328e.

Carretero-Genevrier A., Gich M., Picas L., Sanchez C. et Rodriguez-Carvajal J., «Chiral habit selection on nanostructured epitaxial quartz films », Faraday Discussions, 179, 2015, 227-233, DOI : 10.1039/c4fd00266k.

Fernandez C., Sassoye C., Flores N., Escalona N., Gaigneaux E.M., Sanchez C. et RUIZ P., «Insights in the mechanism of deposition and growth of RuO2 colloidal nanoparticles over alumina. Implications on the activity for ammonia synthesis », Applied Catalysis a-General, 502, 2015, 48-56, DOI : 10.1016/j.apcata.2015.05.023.

\section{Publications vers un public élargi}

SANCHEZ C., « Chimie des matériaux hybrides », L'annuaire du Collège de France. Cours et travaux, $\mathrm{n}^{\circ} 114,2015,241-270$; en ligne : https://annuaire-cdf.revues.org/11897.

\section{BREVETS PUBLIÉS}

Élaboration de polymères nanocomposites par des précurseurs sol-gel en voie non hydrolytique assistée par micro-ondes, S. Delalande ; C. Hoffman ; C. Sanchez ; L. Rozes ; L. Nicole, Peugeot Citroën Automobiles SA; université Pierre et Marie Curie - UPMC; Centre national de la recherche scientifique - CNRS ; Collège de France, $\mathrm{N}^{0}$ de dépôt : FR 1462405 (2014).

Fluoropolymer film, S. Rose ; C. Laberty ; C. Sanchez ; J. Abuseleme, Solvay ; université Pierre et Marie Curie - UPMC ; Centre national de la recherche scientifique - CNRS ; Collège de France, EP application No. 14307146.2 (2014).

New catalyst comprising a carrier, bromine and nickel metal nanoparticles, useful for a selective hydrogenation of $2 \mathrm{C}$ polyunsaturated hydrocarbon charge, C. Thomazeau; J. Aguilhon; O. Durupthy; C. Boissiere; C. Sanchez, IFP ENERGIES NOUVELLES, FR2994661 (2014). 
Procédé de préparation d'une solution colloïdale de nanoparticules d'un oxyde de vanadium ou d'un oxyde de vanadium et d'yttrium, dopé par de l'europium, et utilisations de ladite solution, N. Duée, C. Ambard, K. Valle, D. Portehault, C. Sanchez, YD 15903, Dépôt prioritaire FR 1461067 (2015)

Matériau nanostructuré de bore amorphe, D. Portehault, G. Gouget, C. Gervais-Stary, C. Sanchez, France, date de dépôt : 25/06/2015, numéro de dépôt : 15 55878, déposant(s) : CNRS, Collège de France, UPMC.

\section{PRIX ET DISTINCTIONS}

Récipiendaire du grand prix ISGS (International Sol-Gel Society) 2015, The Life Achievement Award, Kyoto, Japon, septembre 2015. 